\title{
Performance Enhancement for Conventional Tightly Coupled INS/DVL Navigation System Using Regeneration of Partial DVL Measurements
}

\author{
Taesuk Yoo $\mathbb{D}^{1},{ }^{1}$ Moonhwan Kim, ${ }^{1}$ Seonil Yoon, ${ }^{2}$ and Daejoong Kim ${ }^{1}$ \\ ${ }^{1}$ Maritime R\&D Center LIG Nex1 Co., Ltd, Gyeonggi-do 463-300, Republic of Korea \\ ${ }^{2}$ R\&D Planning Team LIG Nex1 Co., Ltd, Gyeonggi-do 463-300, Republic of Korea \\ Correspondence should be addressed to Taesuk Yoo; taesuk.yoo@lignex1.com
}

Received 15 September 2019; Accepted 21 December 2019; Published 11 January 2020

Academic Editor: Valerie Renaudin

Copyright () 2020 Taesuk Yoo et al. This is an open access article distributed under the Creative Commons Attribution License, which permits unrestricted use, distribution, and reproduction in any medium, provided the original work is properly cited.

\begin{abstract}
Inertial navigation systems/Doppler velocity log (INS/DVL) integrated navigation systems are widely used in underwater environments where GPS is unavailable. An INS/DVL integrated navigation system is generally loosely coupled; however, this does not work if any of the DVL transducers do not work. If a system is tightly coupled, velocity error can be estimated with fair accuracy even if some of the transducers fail. However, despite the robustness of a tightly coupled system compared to a loosely coupled one, velocity error estimation accuracy of the former decreases as the number of faulty transducers increases. Therefore, this paper proposes an INS/DVL/revolutions per minute (RPM) integrated navigation filter designed to improve the performance of conventional tightly coupled integrated systems by estimating data from faulty transducers using RPM data. Two salient features of the proposed filter are (1) estimating RPM data accounting for error from the effect of tidal currents and (2) continuous estimation of error in RPM data by selectively converting only the measurements of faulty transducers. The performance of the proposed filter was first verified using Monte Carlo numerical simulations with the analysis range set to 1 standard deviation $(1 \sigma, 68 \%)$ and then with real sea test measurement data.
\end{abstract}

\section{Introduction}

Inertial measurement units (IMUs) and Doppler velocity logs (DVLs) are standard sensors in integrated underwater navigation systems. An IMU measures acceleration and angular velocity using accelerometers and gyroscopes that measure specific force and rotation, respectively, which allows the calculation of navigation information (position, velocity, and attitude) without influence from the environment. However, these IMU estimates are accurate only for a short time range because of the accumulation of navigation errors over time; these are magnified by the integration process [1]. To overcome this drawback, several studies have been conducted to establish methods to correct navigation information using external sensors such as DVLs.

A DVL is used to measure the velocity of an underwater vehicle using the Doppler effect by observing the shift in frequency between an acoustic signal transmitted from the underwater vehicle and its echo signal reflected from the seabed. In a DVL, four transducers are aligned in a Janus configuration, whereby the velocity measured at each transducer is transformed into the three-axis velocity of the vehicle using a coordinate transformation matrix [2].

In general, the velocity correction algorithm of a DVLaided inertial navigation system (INS) employs a loosely coupled approach that uses the body frame velocities calculated by the DVL as the input to the Kalman filter. There has been more research on loosely coupled approaches than tightly coupled ones, and these have focused on designing and verifying navigation filters based mostly on the extended Kalman filter [3-6]. Recent design and verification studies have also used other types of filters such as the unscented Kalman filter and cubature Kalman filter [7-9]. A loosely coupled approach has a design advantage due to the 
simplicity of its measurements, but its accuracy in measuring body frame velocities is greatly impaired if any of the DVL transducer signals cannot be received. To overcome this drawback, various filter designs have also been proposed that use sensors other than DVL or apply a set of constraints [10-13].

Different tightly coupled navigation filters have been designed over the past few years [14, 15]. A tightly coupled system uses the velocity measured by each transducer, not the velocity from the body frame transformed from filter measurements. Even if one or more of the four transducers fail, the consequent velocity error in the INS can be fairly compensated for by the velocity information from the remaining transducers. However, despite its robustness compared to a loosely coupled system, the accuracy of the velocity error estimation of a tightly coupled system decreases as the number of faulty transducers increases.

Revolutions per minute (RPM) data can be used to correct velocity errors in INSs in underwater environments. The relationship between RPM data and the cruising speed of an underwater vehicle can be estimated using a linear equation. This can be derived from a six degrees of freedom (6DOF) model or test results, and accurate velocity information can be obtained under ideal circumstances. However, RPM data alone cannot compensate for the effect of tidal currents, which is an underwater characteristic that must be considered.

In this study, an INS/DVL/RPM integrated navigation filter is designed with the aim of improving the performance of the conventional tightly coupled approach by estimating the information from the faulty transducer using RPM information. The RPM error factor is estimated during the normal operation of the DVL and velocity is calculated in parallel such that the main focus is on estimating the RPM while accounting for the tidal current error factor. If any of the DVL transducers fail during navigation, filter measurements are transformed into RPM and the main focus shifts to transforming the velocity of a faulty transducer instead of the overall velocity. Consequently, filter measurements are based both on the values measured by the normally functioning DVL transducers and the estimated RPM. This method draws on the advantages of the tightly coupled approach so that RPM errors can be estimated continuously using the RPM values measured accurately prior to the transducer failure and the values measured by the normally functioning transducers. In the loosely coupled approach, it is possible to estimate the RPM error factor prior to transducer failure; however, if a failure occurs, then all the measured values must be converted into RPM, meaning that the RPM error factor can no longer be estimated.

The performance of the proposed filter was analyzed via Monte Carlo simulations. The scope of the analysis was comparing the performance of the conventional and proposed tightly coupled methods under the conditions in which two or three DVL transducers became faulty and the presence or absence of the effect of tidal currents. Each scenario was simulated for 100 iterations and the results were analyzed based on standard deviation $(1 \sigma)$. Assuming a normal distribution of the sample, $68 \%$ of all data fall within $1 \sigma$ of the mean. Finally, the performance of the proposed filter was verified using data obtained in real sea tests.

\section{Conventional Method: INS/DVL Integrated Navigation System}

Figure 1 presents the typical structures of loosely coupled (Figure 1(a)) and tightly coupled (Figure 1(b)) INS/DVL integrated navigation systems. The differences between these systems lie in their respective measurement models, whereby velocity errors are estimated along the three axes of the body frame and the four axes of the DVL frame, respectively.

2.1. System Models. Equations (1) and (2) are differential equations for calculating the velocity and attitude errors incurred in the strapdown inertial navigation system. They are standard equations and their derivation processes [1] are therefore omitted here.

$$
\begin{aligned}
\delta \dot{v}_{i}^{n}= & {\left[f_{i}^{n} \times\right] \phi_{i}^{n}+C_{b}^{n} \delta f_{k}^{b}-\left(2 w_{i e, i}^{n}+w_{e n, i}^{n}\right) } \\
& \times \delta v_{i}^{n}(i \in n, e, d k \in x, y, z), \\
\dot{\phi}_{i}^{n}= & -w_{\mathrm{in}, i}^{n} \times \phi-C_{b}^{n} \delta w_{i b, k}^{b}(i \in n, e, d k \in x, y, z) .
\end{aligned}
$$

In these equations, $\delta v_{i}^{n}$ is velocity error, $\left[f_{i}^{n} \times\right]$ is the skew-symmetric matrix of the specific force in the navigation frame, $\varphi_{i}^{n}$ is the attitude error, $C_{b}^{n}$ is the attitude transformation matrix, $\delta f_{k}^{b}$ is the accelerometer error, $2 w_{i e, i}^{n}$, $w_{e n, i}^{n}$, and $w_{\mathrm{in}, i}^{n}$ are the angular velocity of the rotation of the Earth and transport rate components, and $\delta w_{i b, k}^{b}$ is the gyro error.

The system model is constituted as a time-varying linear system by combining the differential equations for velocity and attitude errors obtained using Equations (1) and (2) can be defined as follows:

$$
\delta \dot{x}(t)=F(t) \delta x(t)+w(t), w(t) \sim N(0, Q(t))
$$

Equation (3) can then be transformed to its discrete form, expressed as below:

$$
\begin{aligned}
\delta x_{k+1} & =\Phi_{k} \delta x_{k}+w_{k}=e^{F(k) d t} \delta x_{k}+w_{k} \\
& \cong\left(I+F(k) \mathrm{dt}+F(k)^{2} \mathrm{dt}^{2} / 2\right) \delta x_{k}+w_{k},
\end{aligned}
$$

where $\mathrm{dt}$ is the propagation timestep of the state variable. $12^{\text {th }}$-order state variable can be modeled as follows:

$$
\delta x=\left[\delta v_{i, \text { ins }}^{n} \varphi_{i}^{n} \nabla_{k} \varepsilon_{k}\right]^{T}(i \in n, e, d k \in x, y, z)
$$

where $\delta v_{i, \text { ins }}^{n}$ is the velocity error, $\phi_{i}^{n}$ is the attitude order, $\nabla_{k}$ is the acceleration bias, and $\varepsilon_{k}$ is the gyro bias. Using 


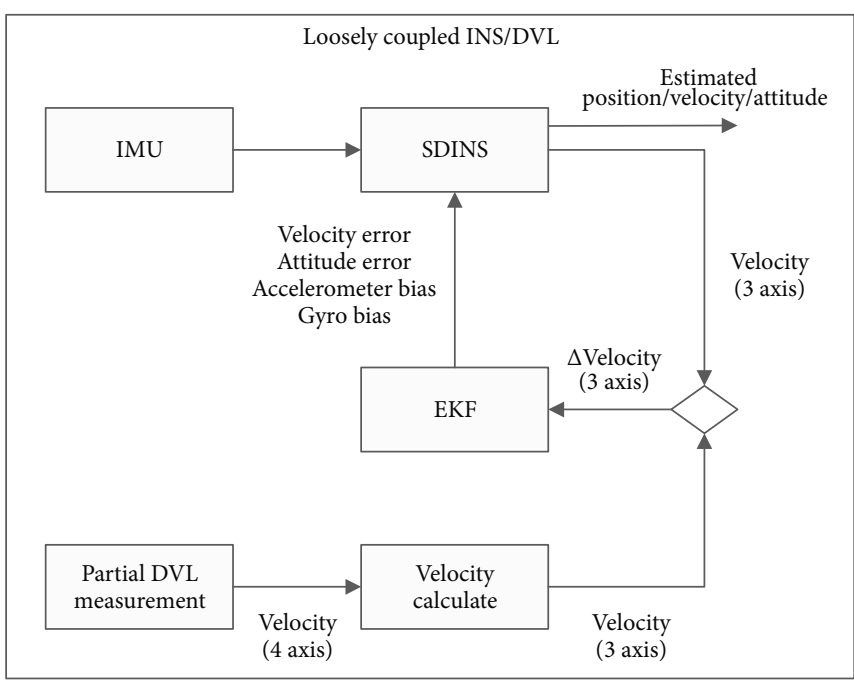

(a)

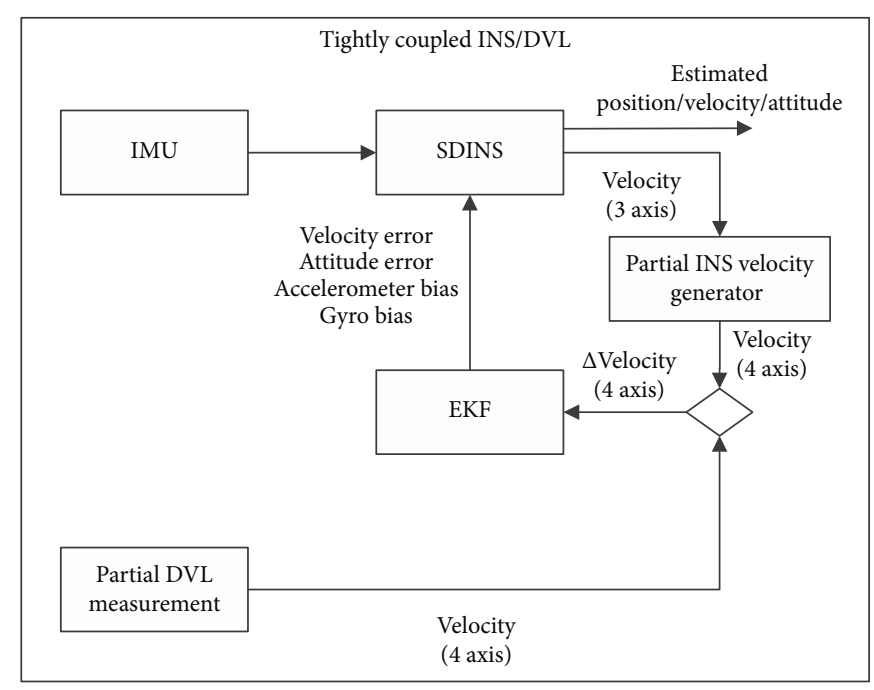

(b)

FIGURE 1: General structure of loosely/tightly coupled INS/DVL.

Equations (1) and (2), system error model $F(t)$ can be determined as below:

$$
\begin{aligned}
& F(t)=\left[\begin{array}{cccc}
F_{11} & {\left[C_{b}^{n} f^{b} \times\right]} & C_{b}^{n} & 0_{3 \times 3} \\
F_{21} & F_{22} & 0_{3 \times 3} & -C_{b}^{n} \\
0_{3 \times 3} & 0_{3 \times 3} & 0_{3 \times 3} & 0_{3 \times 3} \\
0_{3 \times 3} & 0_{3 \times 3} & 0_{3 \times 3} & 0_{3 \times 3}
\end{array}\right] \\
& F_{11}=\left[\begin{array}{ccc}
\frac{v_{d}}{R_{m}+h} & 2 w_{i e, d}^{n}+2 w_{e n, d}^{n} & -w_{e n, e}^{n} \\
-2 w_{i e, d}^{n}-w_{e n, d}^{n} & \frac{v_{n} \tan (L)+v_{d}}{R_{t}+h} & 2 w_{i e, n}^{n}+w_{e n, n}^{n} \\
2 w_{e n, e}^{n} & -2 w_{i e, n}^{n}-2 w_{e n, n}^{n} & 0
\end{array}\right] \text {, } \\
& F_{21}=\left[\begin{array}{ccc}
0 & \frac{1}{R_{t}+h} & 0 \\
-\frac{1}{R_{m}+h} & 0 & 0 \\
0 & -\frac{\tan (L)}{R_{t}+h} & 0
\end{array}\right] \text {, } \\
& F_{22}=\left[\begin{array}{ccc}
0 & w_{i e, d}^{n}+w_{e n, d}^{n} & -w_{e n, e}^{n} \\
-w_{i e, d}^{n}-w_{e n, d}^{n} & 0 & w_{i e, n}^{n}+w_{e n, n}^{n} \\
w_{e n, e}^{n} & -w_{i e, n}^{n}-w_{e n, n}^{n} & 0
\end{array}\right] .
\end{aligned}
$$

In this matrix, $L$ is latitude and $R_{m}$ and $R_{t}$ are defined as follows:

$$
R_{m}=\frac{R_{0}\left(1-e^{2}\right)}{\left(1-e^{2} \sin ^{2} L\right)^{3 / 2}}, R_{t}=\frac{R_{0}}{\left(1-e^{2} \sin ^{2} L\right)^{1 / 2}}
$$

where $R_{0}, e$, and $L$ are the Earth's equatorial radius, Earth's ellipticity, and latitude, respectively.

\subsection{Measurement Models}

2.2.1. Loosely Coupled Measurement Model. A loosely coupled measurement model explains the velocity errors along the three axes of the navigation frame. Velocity error is the difference between the INS-calculated and DVLmeasured velocity, defined as follows:

$$
v_{i, \mathrm{ins}}^{n}-v_{i, \mathrm{dvl}}^{n}=v_{i, \mathrm{ins}}^{n}-C_{b}^{n} v_{k, \mathrm{dvl}}^{b}(i \in n, e, d k \in x, y, z) .
$$

Before Equation (8) is derived for the error model, Equation (9) is derived using the perturbation method, expressed as below:

$$
\begin{aligned}
v_{i, \mathrm{ins}}^{n} & -v_{i, \mathrm{dvl}}^{n}=v_{i, \mathrm{ins}}^{n}+\delta v_{i, \mathrm{ins}}^{n} \\
& -\left(C_{b}^{n} v_{k, \mathrm{dvl}}^{b}+\delta C_{b}^{n} v_{k, \mathrm{dvl}}^{b}+C_{b}^{n} \delta v_{k, \mathrm{dvl}}^{b}\right)=v_{i, \mathrm{ins}}^{n}+\delta v_{i, \mathrm{ins}}^{n} \\
& -\left(C_{b}^{n} v_{k, \mathrm{dvl}}^{b}+(-\delta \phi \times) C_{b}^{n} v_{k, \mathrm{dvl}}^{b}+C_{b}^{n} \delta v_{k, \mathrm{dvl}}^{b}\right)=v_{i, \mathrm{ins}}^{n}+\delta v_{i, \mathrm{ins}}^{n} \\
& -C_{b}^{n} v_{k, \mathrm{dvl}}^{b}-(-\delta \phi \times) C_{b}^{n} v_{k, \mathrm{dvl}}^{b}-C_{b}^{n} \delta v_{k, \mathrm{dvl}}^{b}=v_{i, \mathrm{ins}}^{n}+\delta v_{i, \mathrm{ins}}^{n} \\
& -C_{b}^{n} v_{k, \mathrm{dvl}}^{b}-\left(C_{b}^{n} v_{k, \mathrm{dvl}}^{b} \times\right) \delta \phi-\delta v_{i, \mathrm{dvl}}^{n}=\delta v_{i, \mathrm{ins}}^{n} \\
& -\left(v_{i, \mathrm{ins}}^{n} \times\right) \delta \phi-\delta v_{i, \mathrm{dvl}}^{n}
\end{aligned}
$$

The measurement matrix $\mathrm{H}$ can therefore be designed as follows:

$$
H=\left[\begin{array}{llll}
I_{3 \times 3} & -\left(v_{i, \text { ins }}^{n} \times\right) & 0_{3 \times 3} & 0_{3 \times 3}
\end{array}\right] .
$$

2.2.2. Tightly Coupled Measurement Model. A tightly coupled measurement model explains the velocity errors along the 
four axes of the DVL frame. The transformation matrix for body frame and DVL frame is as below [2]:

$$
v_{j, \text { ins }}=C_{b}^{d} v_{k, \mathrm{dvl}}^{b}(j \in 1,2,3,4 k \in x, y, z) \text {, }
$$

where $C_{b}^{d}$ is the term for the transformation between the body frame and DVL frame. It is determined according to the following instrumentation setup for DVL:

$$
C_{b}^{d}=\left[\begin{array}{ccc}
\cos \alpha \cos \gamma & \cos \alpha \sin \gamma & \sin \alpha \\
\cos \alpha \sin \gamma & -\cos \alpha \cos \gamma & \sin \alpha \\
-\cos \alpha \cos \gamma & -\cos \alpha \sin \gamma & \sin \alpha \\
-\cos \alpha \sin \gamma & \cos \alpha \cos \gamma & \sin \alpha
\end{array}\right],
$$

where $\alpha$ and $\gamma$ are the tilt angles of the four transducers relative to the sea floor and the cruising direction of the underwater vehicle, respectively. If the DVL is aligned in the $(+)$ form relative to the latter, $\gamma=0^{\circ}$; in the $(x)$ form, $\gamma=45^{\circ}$.

The measurement model represents the real-time difference between the INS-calculated velocity and the DVL-measured velocity, defined as below:

$$
v_{j, \text { ins }}-v_{j, \mathrm{dvl}}=C_{b}^{d} C_{n}^{b} v_{i, \text { ins }}^{n}-v_{j, \mathrm{dvl}}(i \in n, e, d j \in 1,2,3,4) .
$$

Before Equation (13) is derived for the error model, Equation (14) is derived using the perturbation method, expressed as follows:

$$
\begin{aligned}
v_{j, \text { ins }} & -v_{j, \mathrm{dvl}}=C_{b}^{d} C_{n}^{b} v_{i, \mathrm{ins}}^{n}+C_{b}^{d} \delta C_{n}^{b} v_{i, \mathrm{ins}}^{n}+C_{b}^{d} C_{n}^{b} \delta v_{i, \mathrm{ins}}^{n} \\
& -\left(v_{j, d v l}+\delta v_{j, \mathrm{dvl}}\right)=C_{b}^{d} C_{n}^{b} v_{i, \mathrm{ins}}^{n}+C_{b}^{d} C_{n}^{b}(\delta \phi \times) v_{i, \mathrm{ins}}^{n} \\
& +C_{b}^{d} C_{n}^{b} \delta v_{i, \text { ins }}^{n}-\left(v_{j, d v l}+\delta v_{j, \mathrm{dvl}}\right)=C_{b}^{d} C_{n}^{b} v_{i, \mathrm{ins}}^{n} \\
& -C_{b}^{d} C_{n}^{b}\left(v_{i, \mathrm{ins}}^{n} \times\right) \delta \phi+C_{b}^{d} C_{n}^{b} \delta v_{i, \mathrm{ins}}^{n}-v_{j, \mathrm{dvl}} \\
& -\delta v_{j, \mathrm{dvl}}=C_{b}^{d} C_{n}^{b} \delta v_{i, \mathrm{ins}}^{n}-C_{b}^{d} C_{n}^{b}\left(v_{i, \mathrm{ins}}^{n} \times\right) \delta \phi-\delta v_{j, \mathrm{dvl}}
\end{aligned}
$$

The measurement matrix $\mathrm{H}$ can therefore be designed as

$$
H=\left[\begin{array}{llll}
C_{b}^{d} C_{n}^{b} & -C_{b}^{d} C_{n}^{b}\left(v_{i, \text { ins }}^{n} \times\right) & 0_{4 \times 3} & 0_{4 \times 3}
\end{array}\right] .
$$

\section{Proposed Method: Design of Tightly Coupled INS/DVL/RPM Integrated Navigation System}

The main difference between loosely and tightly coupled integration lies in the presence or absence of a DVL transducer failure. The loosely coupled approach does not work if any of the four transducers is faulty, whereas the tightly coupled approach allows velocity error estimation based on the data from the remaining transducers that are operating normally. Although the tightly coupled approach thus outperforms the loosely coupled approach, the accuracy of velocity error estimation under the conditions in which one transducer is faulty state is inevitably lower compared with the prefailure state.

In an attempt to improve the performance of the conventional tightly coupled approach, this study proposes an INS/DVL/RPM integrated navigation filter (Figure 2). The proposed method uses RPM data obtained from the underwater vehicle's motor drive data to estimate the cruising speed in the body frame. Thus, the RPM/velocity relationship can be obtained through a simple estimation of cruising speed combined with a 6DOF motion model or test result analysis. RPM-based velocity estimation is highly accurate under ideal circumstances, expressed as follows:

$$
v_{\mathrm{rpm}}^{b}=\mathrm{sf} \times \mathrm{rpm}
$$

The error model of Equation (16) is derived as below:

$$
v_{\mathrm{rpm}}^{b}+\delta v_{\mathrm{rpm}}^{b}=\mathrm{sf} \times \mathrm{rpm}+\delta \mathrm{sfrpm}+\mathrm{sf} \delta \mathrm{rpm},
$$

where $\delta v_{\mathrm{rpm}}^{b}$ is the RPM-based estimate of the error in cruising direction velocity, which consists of sf error and RPM bias error. The effect of tidal currents as a state variable must be added to this and the whole considered further. The accuracy of RPM-estimated velocity is very high in the absence of tidal currents but decreases under their influence. The accuracy can therefore be improved if the effect of tidal currents can be estimated to compensate for the need to use RPM-estimated velocity. The state variable accounting for the RPM error factor is set as a $17^{\text {th }}$ order state variable as follows:

$$
\begin{array}{r}
\delta x=\left[\begin{array}{lllllll}
\delta v_{i, \mathrm{ins}}^{n} & \varphi_{i}^{n} & \nabla_{k} & \varepsilon_{k} & \delta_{b, \mathrm{rpm}} & \delta_{s f, \mathrm{rpm}} & \delta v_{i, \mathrm{tide}}^{n}
\end{array}\right]^{T} \\
(i \in n, e, d k \in x, y, z),
\end{array}
$$

where $\delta_{b, \text { rpm }}$ is the RPM bias error, $\delta_{\text {sf,rpm }}$ is the RPM scale factor error, and $\delta v_{i, \text { tide }}^{n}$ is the navigation frame velocity error relative to tidal currents. The system error model $F(t)$ can be determined as follows:

$$
F(t)=\left[\begin{array}{ccccccc}
F_{11} & {\left[C_{b}^{n} f^{b} \times\right]} & C_{b}^{n} & 0_{3 \times 3} & 0_{3 \times 1} & 0_{3 \times 1} & 0_{3 \times 3} \\
F_{21} & F_{22} & 0_{3 \times 3} & -C_{b}^{n} & 0_{3 \times 1} & 0_{3 \times 1} & 0_{3 \times 3} \\
0_{3 \times 3} & 0_{3 \times 3} & 0_{3 \times 3} & 0_{3 \times 3} & 0_{3 \times 1} & 0_{3 \times 1} & 0_{3 \times 3} \\
0_{3 \times 3} & 0_{3 \times 3} & 0_{3 \times 3} & 0_{3 \times 3} & 0_{3 \times 1} & 0_{3 \times 1} & 0_{3 \times 3} \\
0_{1 \times 3} & 0_{1 \times 3} & 0_{1 \times 3} & 0_{1 \times 3} & 0_{1 \times 1} & 0_{1 \times 1} & 0_{1 \times 3} \\
0_{1 \times 3} & 0_{1 \times 3} & 0_{1 \times 3} & 0_{1 \times 3} & 0_{1 \times 1} & 0_{1 \times 1} & 0_{1 \times 3} \\
0_{3 \times 3} & 0_{3 \times 3} & 0_{3 \times 3} & 0_{3 \times 3} & 0_{3 \times 1} & 0_{3 \times 1} & 0_{3 \times 3}
\end{array}\right],
$$




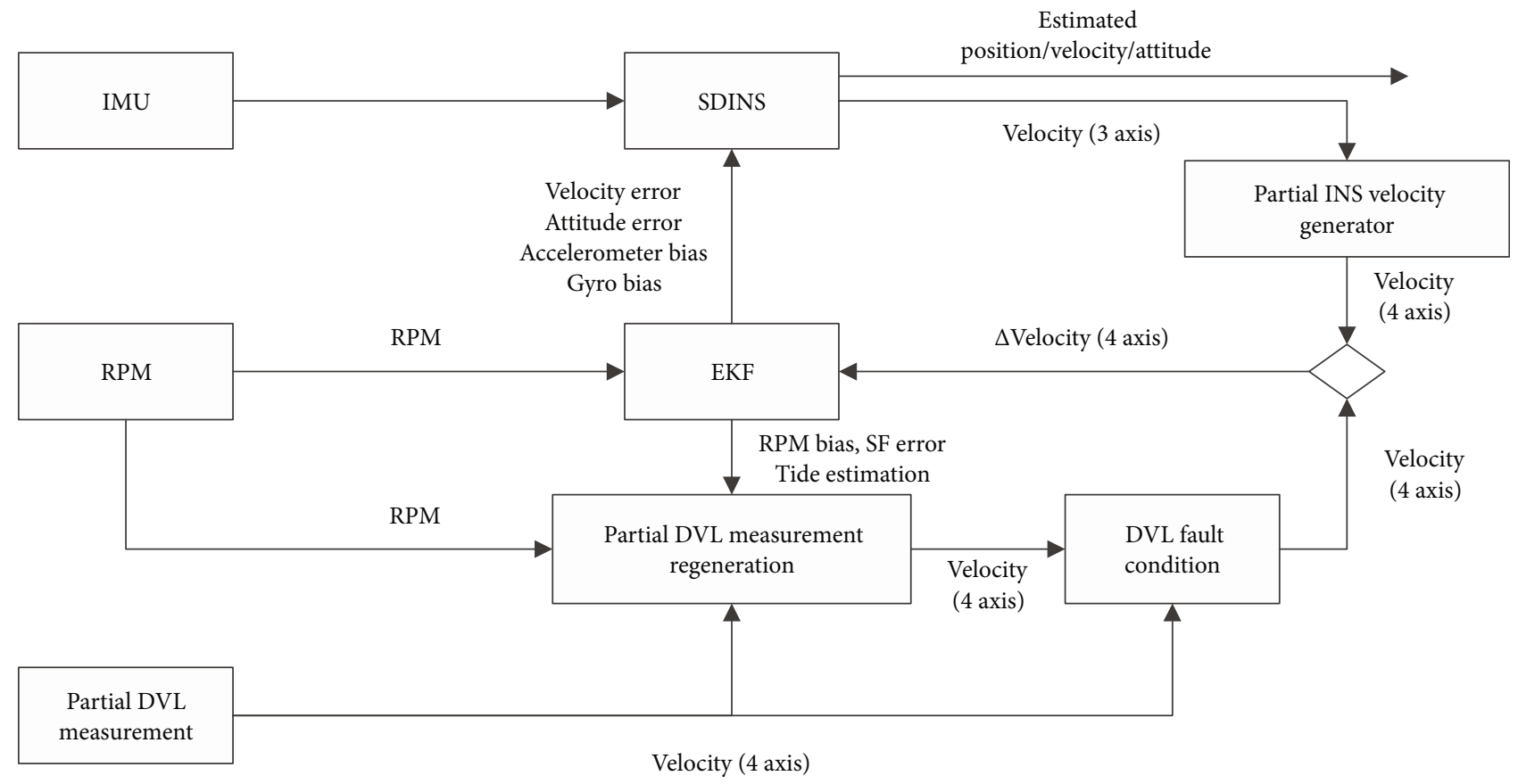

FIGURE 2: Structure of the proposed tightly coupled INS/DVL/RPM integrated navigation system.

where $F_{11}, F_{21}$, and $F_{22}$ are identical to those in Equation (6). A measurement model that does not consider the effect of tidal currents can be designed as below:

$$
v_{x}^{b}-v_{\mathrm{rpm}}^{b}=C_{n(1,1: 3)}^{b} v_{i, \mathrm{ins}}^{n}-(\mathrm{sf} \times \mathrm{rpm})(i \in n, e, d)
$$

Before Equation (20) can be derived for the error model, Equation (21) is derived using the perturbation method, expressed as follows:

$$
\begin{aligned}
v_{x}^{b}-v_{\mathrm{rpm}}^{b}= & C_{n(1,1: 3)}^{b} v_{i, \mathrm{ins}}^{n}+\delta C_{n(1,1: 3)}^{b} v_{i, \mathrm{ins}}^{n}+C_{n(1,1: 3)}^{b} \delta v_{i, \mathrm{ins}}^{n} \\
& -\left(\operatorname{sfrpm}+\operatorname{rpm} \delta_{\mathrm{sf}, \mathrm{rpm}}+s f \delta_{b, \mathrm{rpm}}\right)=C_{n(1,1: 3)}^{b} v_{i, \mathrm{ins}}^{n} \\
& +C_{n(1,1: 3)}^{b}(\delta \phi \times) v_{i, \mathrm{ins}}^{n}+C_{n(1,1: 3)}^{b} \delta v_{i, \mathrm{ins}}^{n} \\
& -\left(\operatorname{sfrpm}+\operatorname{rpm} \delta_{\text {sf }, \mathrm{rpm}}+\operatorname{sf} \delta_{b, \mathrm{rpm}}\right)=C_{n(1,1: 3)}^{b} v_{i, \mathrm{ins}}^{n} \\
& -C_{n(1,1: 3)}^{b}\left(v_{i, \mathrm{ins}}^{n} \times\right) \delta \phi+C_{n(1,1: 3)}^{b} \delta v_{i, \mathrm{ins}}^{n}-\operatorname{sfrpm} \\
& -\operatorname{rpm} \delta_{\mathrm{sf}, \mathrm{rpm}}-\operatorname{sf} \delta_{b, \mathrm{rpm}}=C_{n(1,1: 3)}^{b} \delta v_{i, \mathrm{ins}}^{n} \\
& -C_{n(1,1: 3)}^{b}\left(v_{i, \mathrm{ins}}^{n} \times\right) \delta \phi-\operatorname{sf} \delta_{b, \mathrm{rpm}}-\operatorname{rpm} \delta_{s f, \mathrm{rpm}} .
\end{aligned}
$$

Assuming the absence of the effect of tidal currents, the cruising speed of an underwater vehicle can be estimated accurately using RPM with the model defined by Equation (21). If any of the four DVL transducers is faulty, RPM data can be used instead of DVL to estimate velocity. The accuracy of the velocity error estimation using Equation (21) is reduced in the presence of tidal currents. It is therefore essential to compensate for the effect of tidal currents when RPM-based velocity estimation is performed. The corresponding measurement model can be determined as follows:

$$
v_{x}^{b}-v_{\mathrm{rpm}}^{b}=C_{n(1,1: 3)}^{b}\left(v_{i, \mathrm{ins}}^{n}-v_{i, \mathrm{ide}}^{n}\right)-(\mathrm{sf} \times \mathrm{rpm})(i \in n, e, d) .
$$

Before Equation (22) can be derived for error model, Equation (23) is derived using the perturbation method, expressed as below:

$$
\begin{aligned}
v_{x}^{b}-v_{\mathrm{rpm}}^{b}= & C_{n(1,1: 3)}^{b} v_{i, \text { ins }}^{n}+\delta C_{n(1,1: 3)}^{b} v_{i, \text { ins }}^{n}+C_{n(1,1: 3)}^{b} \delta v_{i, \text { ins }}^{n} \\
& -\left(C_{n(1,1: 3)}^{b} v_{i, \text { tide }}^{n}+\delta C_{n(1,1: 3)}^{b} v_{i, \text { tide }}^{n}+C_{n(1,1: 3)}^{b} \delta v_{i, \text { tide }}^{n}\right) \\
& -\left(\operatorname{sfrpm}+\operatorname{rpm} \delta_{s f, \mathrm{rpm}}+s f \delta_{b, \mathrm{rpm}}\right)=C_{n(1,1: 3)}^{b} v_{i, \text { ins }}^{n} \\
& +C_{n(1,1: 3)}^{b}(\delta \phi \times) v_{i, \text { ins }}^{n}+C_{n(1,1: 3)}^{b} \delta v_{i, \text { ins }}^{n} \\
& -\left(C_{n(1,1: 3)}^{b} v_{i, \text { tide }}^{n}+C_{n(1,1: 3)}^{b}(\delta \phi \times) v_{i, \text { tide }}^{n}\right. \\
& \left.+C_{n(1,1: 3)}^{b} \delta v_{i, \text { tide }}^{n}\right)-\left(\operatorname{sfrpm}+\operatorname{rpm} \delta_{\text {sf,rpm }}+\operatorname{sf} \delta_{b, \text { rpm }}\right) \\
= & C_{n(1,1: 3)}^{b} v_{i, \text { ins }}^{n}-C_{n(1,1: 3)}^{b}\left(v_{i, \text { ins }}^{n} \times\right) \delta \phi+C_{n(1,1: 3)}^{b} \delta v_{i, \text { ins }}^{n} \\
& -C_{n(1,1: 3)}^{b} v_{i, \text { tide }}^{n}+C_{n(1,1: 3)}^{b}\left(v_{i, \text { tide }}^{n} \times\right) \delta \phi-C_{n(1,1: 3)}^{b} \delta v_{i, \text { tide }}^{n} \\
& -\operatorname{sfrpm}-\operatorname{rpm} \delta_{\text {sf,rpm }}-\operatorname{sf} \delta_{b, \mathrm{rpm}}=C_{n(1,1: 3)}^{b} \delta v_{i, \text { ins }}^{n} \\
& -C_{n(1,1: 3)}^{b}\left(\left[v_{i, \text { ins }}^{n} \times\right]-\left[v_{i, \text { tide }}^{n} \times\right]\right) \delta \phi-\operatorname{sf} \delta_{b, r \mathrm{pm}} \\
& -\operatorname{rpm} \delta_{\text {sf }, r \mathrm{pm}}-C_{n(1,1: 3)}^{b} \delta v_{i, \text { tide }}^{n} .
\end{aligned}
$$




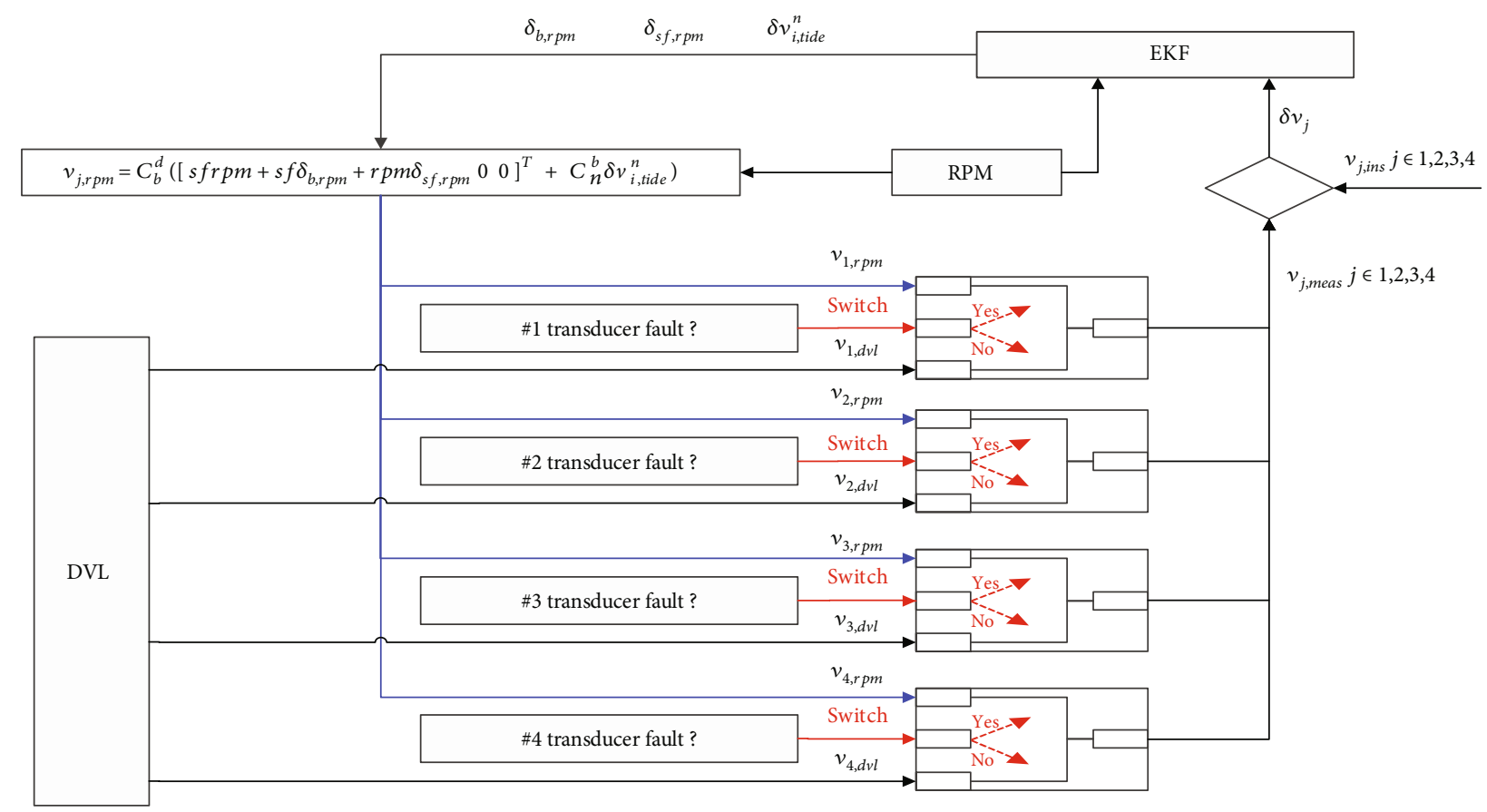

FIGURe 3: Regeneration structure of partial DVL measurement.

The measurement matrix $\mathrm{H}$ can thus be designed as follows:

$$
H=\left[\begin{array}{ccccccc}
C_{b}^{d} C_{n}^{b} & -C_{b}^{d} C_{n}^{b}\left(v_{i, \text { ins }}^{n} \times\right) & 0_{4 \times 3} & 0_{4 \times 3} & 0_{4 \times 1} & 0_{4 \times 1} & 0_{4 \times 3} \\
C_{n(1,1: 3)}^{b} & -C_{n(1,1: 3)}^{b}\left(\left[v_{i, \text { ins }}^{n} \times\right]-\left[v_{i, \text { tide }}^{n} \times\right]\right) & 0_{1 \times 3} & 0_{1 \times 3} & -\mathrm{sf} & -\mathrm{rpm} & -C_{n(1,1: 3)}^{b}
\end{array}\right]
$$

Finally, velocity estimation taking account of RPM bias, scale factor errors, and the effect of tidal currents can be expressed as below:

$$
\begin{array}{r}
v_{k, \mathrm{rpm}}^{b}=\left[\begin{array}{lll}
\operatorname{sfrpm}+\operatorname{sf} \delta_{b, \mathrm{rpm}}+\operatorname{rpm} \delta_{\mathrm{sf}, \mathrm{rpm}} & 0 & 0
\end{array}\right]^{T}+C_{n}^{b} \delta v_{i, \text { tide }}^{n} \\
(i \in n, e, d \quad k \in x, y, z) .
\end{array}
$$

The estimated RPM-based velocity of the body frame can be transformed to the DVL frame as follows:

$$
v_{j, \mathrm{rpm}}=C_{b}^{d} v_{k, \mathrm{rpm}}^{b} \quad(j \in 1,2,3,4 \quad k \in x, y, z) .
$$

In the event of DVL failure, the RPM-based velocity can be estimated in real time by taking account of the effects of tidal currents and thus compensating for the use of Equation (27). For example, if DVL transducers \#2 and \#3 are faulty, the data from transducers \#1 and \#4 are used for RPM- based velocity estimation while transducers \#2 and \#3 are used for DVL-measured velocity, as below:

$$
\text { if } j^{\text {th }} \text { DVL fail, then } v_{j, \mathrm{dvl}}=v_{j, \mathrm{rpm}} \quad j \in 1,2,3,4 \text {. }
$$

The main advantage of the proposed filter is the accuracy of its velocity estimation that takes tidal currents into account by combining intact DVL and RPM data. This draws on the advantages of the tightly coupled approach while overcoming the drawback associated with the use of the conventional tightly coupled approach that considers only RPM data as measurement values in the event of DVL failure. This is because the latter makes it impossible to perform RPMbased velocity error estimation, thus making it no better than the loosely coupled approach. Figure 3 presents a schematic representation of the structure expressed by Equation (27).

\section{Numerical Simulations}

4.1. Overview. We performed Monte Carlo simulations to analyze the performance of the proposed filter. The scope 


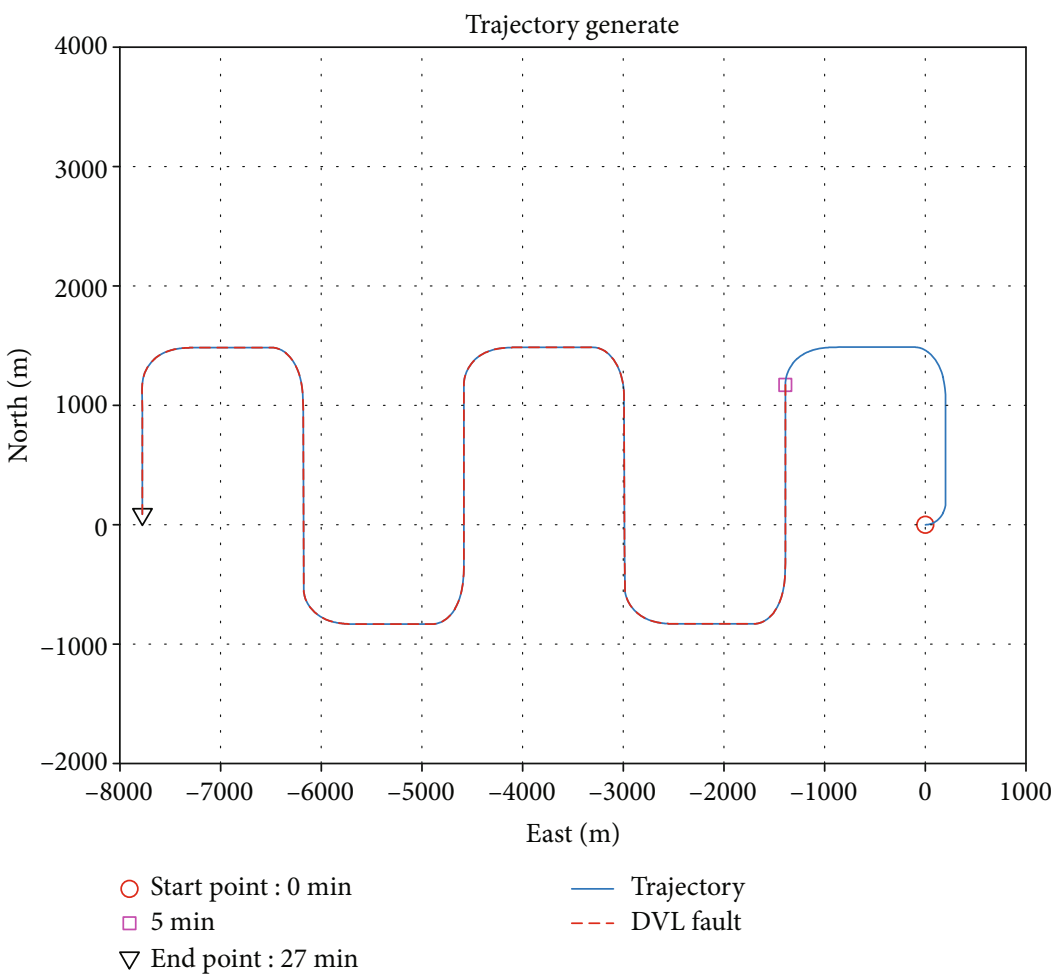

FIGURE 4: Conditions for generating and simulating underwater vehicle trajectory.

of the analysis was a comparison of the performance of the conventional and proposed tightly coupled methods under the conditions in which tidal currents were either present or absent and in which two or three DVL transducers were faulty. Each scenario was simulated for 100 iterations, and the simulation results were analyzed for one standard deviation of the mean $(1 \sigma, 68 \%)$.

Simulation trajectories were generated for analysis as shown in Figure 4. The total time taken for navigation was $27 \mathrm{~min}$ and the cruising speed was $12 \mathrm{~m} / \mathrm{s}$. The onset of DVL failure was set for $5 \mathrm{~min}$ after navigation began. The sce- nario in which two transducers are faulty involve transducers $\# 1$ and $\# 2$, and while that three faulty transducers involves transducers \#1, \#2, and \#3. The IMU was modeled with the trajectories generated using the reverse inertial navigation method. The signals generated for the IMU model took bias, scale factor and misalignment errors, and noise into account whereby performing noise modeling as a $1^{\text {st }}$-order Markov process. Signals for the DVL frame were generated by taking scale factor error, bias, and noise into account. Equations (28), (29), and (30) represent models for the gyroscope, accelerometer, and DVL, respectively.

$$
\begin{aligned}
w_{k, \mathrm{ib}}^{b} & =\left(M_{\mathrm{DCM}} \times \tilde{w}_{k, \mathrm{ib}}^{b}\right) \times(1+\mathrm{sf})+(1-\beta \times \mathrm{dt}) \times b_{k, \text { repeatability }}+b_{k, \text { stability }} \times \sqrt{2 \beta} \times \mathrm{dt} \quad(k \in x, y, z), \\
f_{k}^{b} & =\left(M_{\mathrm{DCM}} \times \tilde{f}_{k}^{b}\right) \times(1+\mathrm{sf})+(1-\beta \times \mathrm{dt}) \times b_{k, \text { repeatability }}+b_{k, \text { stability }} \times \sqrt{2 \beta} \times \mathrm{dt} \quad(i \in x, y, z), \\
v_{j, \mathrm{dvl}} & =C_{b}^{d}\left((1+\mathrm{sf})\left(\tilde{v}_{k, \mathrm{dvl}}\right)\right)+b_{j}+w_{j} \quad(j \in 1,2,3,4 k \in x, y, z) .
\end{aligned}
$$

In these equations, $M_{\mathrm{DCM}}$ is the misalignment, $\mathrm{sf}$ is the scale factor error, $\mathrm{dt}$ is the computation cycle, $b_{k \text {,repeatability }}$ is the bias repeatability, $b_{k, \text { stability }}$ is the bias stability, and $\beta$ is the Markov process time constant. As the DVL transducer alignment was assumed to be in the $(+)$ direction relative to the cruising direction, the value of $C_{b}^{d} \gamma=0$. To determine the effect of tidal currents, tide generation was performed in the navigation frame. Each data point was subjected to Monte Carlo simulations for 100 iterations, as shown in Figure 5. Tidal currents with a flow rate range of -2 to $2 \mathrm{~m} / \mathrm{s}$ $(1 \sigma)$ were generated randomly for analysis, as shown in Figure 5. 

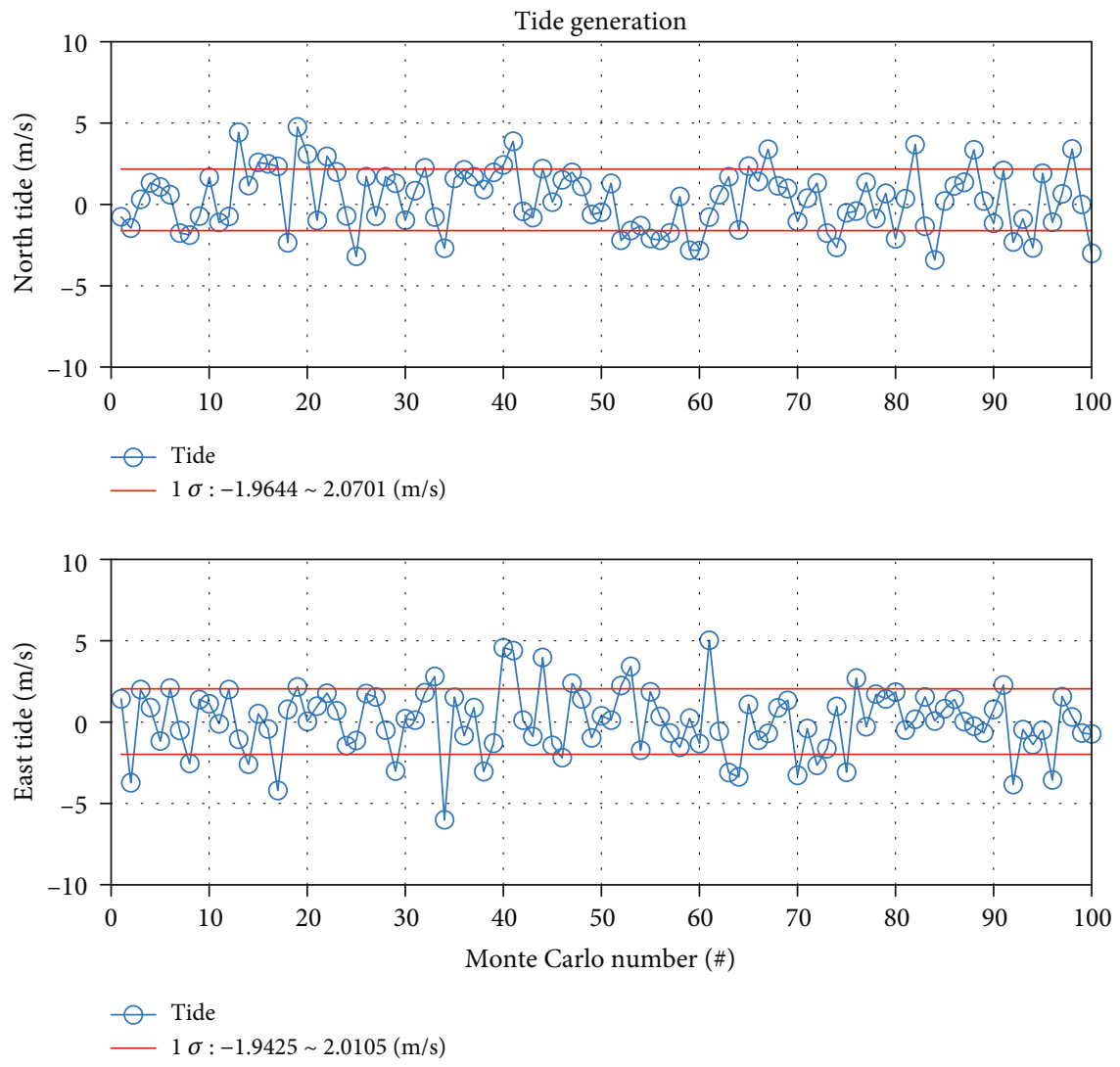

FIGURE 5: Tide generation in the navigation frame.

\subsection{Performance Analysis}

4.2.1. Conditions in which Tides Were Absent. Figure 6 presents the results of velocity and position errors in the scenario in which two transducers are faulty. The blue and red lines in Figure $6(\mathrm{a})$ represent the $1 \sigma$ values of the conventional and proposed tightly coupled methods, respectively. A comparison of these shows that the proposed method outperformed the conventional method in terms of velocity error estimation when the transducer failure occurred after $300 \mathrm{~s}$. This improved performance can be ascribed to the additional RPM error factor that is estimated to compensate for the transducer failure. The blue line shows that the conventional tightly coupled method could estimate velocity error to some extent under the conditions tested, unlike the loosely coupled approach, which would not have been able to do so and would thus have produced a sudden error in velocity. Figures 6(b) and 6(c) represent the velocity and position error profiles, respectively. Figure 7 presents the analysis results for the scenario in which three transducers are faulty. As the number of faulty transducers increases, the velocity error estimation of the conventional tightly coupled method decreases. In contrast, the proposed method maintains its estimation accuracy irrespective of the number of faulty transducers. Thus, there is no significant difference in the position error profile. This confirms that the RPM-based velocity estimation is close to the ideal value in nontidal conditions, where the proposed method is more robust than the conventional tightly coupled method.
4.2.2. Conditions in Which Tides Were Present. The superiority of the proposed filter is manifested more clearly in the presence of tidal currents. Given that the reference frame used for tide error modeling is the navigation frame, the estimated velocity varies depending on the yaw angle. The trajectory in Figure 4 indicates a 90-degree turn immediately after navigation begins for rapid tide error estimation. Figure 8 presents the results of tide error estimation in which the tide error is shown to converge towards zero over time. The RPM velocity error can be compensated for using the estimated tide error according to Equation (25). Figure 9 illustrates result of the velocity and position error analysis for the scenario in which two transducers become faulty and tidal currents are present. It shows that the proposed method outperforms the conventional tightly coupled method in velocity error estimation in this scenario. The other scenario, in which three transducers are faulty and tidal currents are present, yields the same result; this is shown in Figure 10. The proposed filter estimates velocity error regardless of the number of faulty transducers whereas the accuracy of conventional tightly coupled method decreases as the number of faulty transducers increases.

4.2.3. Overall Analysis. Figure 11 presents the final position error profiles. The comparison of the position errors between the conventional and proposed tightly coupled methods in four simulation scenarios shows that in the conventional tightly coupled method, position error increases as the number of faulty transducers increases, but the proposed filter 

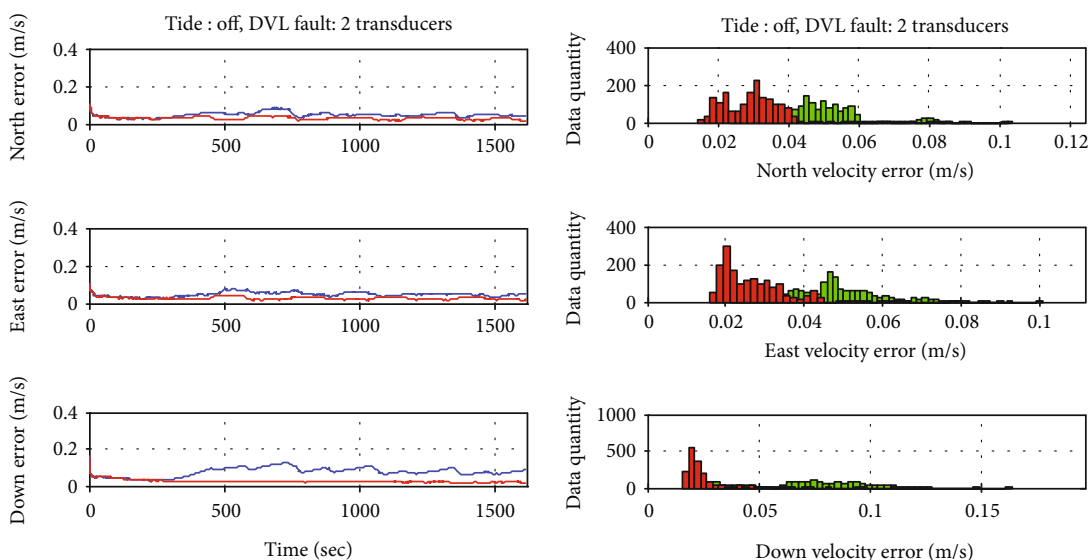

- Conventional TC : $1 \sigma$

$\square$ Conventional TC

$\square$ Proposed TC

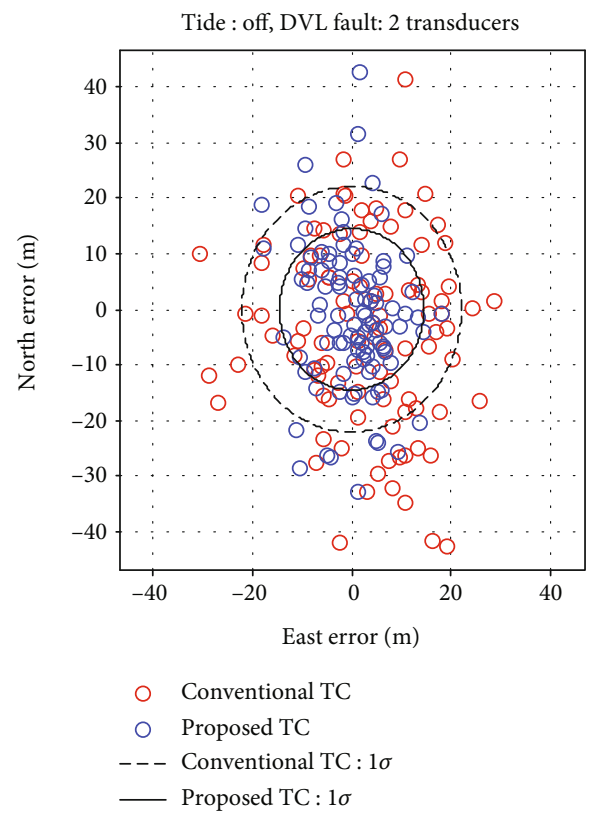

(a)

(b)

(c)

Figure 6: Result of Monte Carlo simulation (tide: off, DVL fault: two transducers).
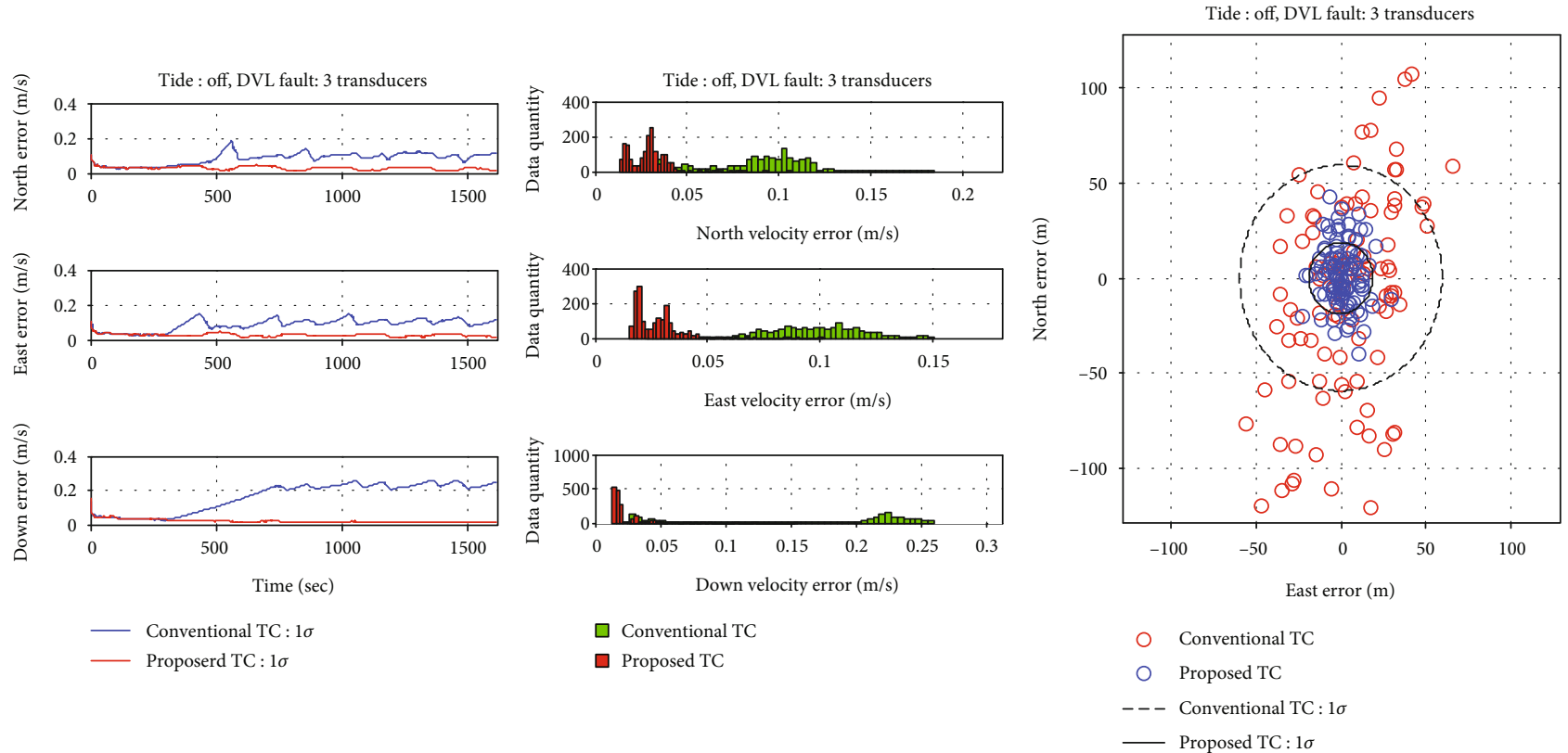

FIGURE 7: Result of Monte Carlo simulation (tide: off, DVL fault: three transducers).

estimated position error is consistently irrespective of the number of faulty transducers. In the absence of tidal currents, consistent estimation is possible because RPM is close to an ideal state. However, the superiority of the proposed method is demonstrated more clearly in the presence of tidal currents. The lack of data on the direction and magnitude of tidal currents makes consistent position error estimation a great challenge unless tide error is estimated with accuracy. The proposed filter was verified as having overcome that challenge by estimating tide error with a high accuracy.
Table 1 outlines the results of position error estimation in each scenario simulated.

\section{Verification with Sea Test Data}

For the final verification of the performance of the proposed filter, we performed an analysis based on real sea test data. The duration of the navigation in this analysis is $20 \mathrm{~min}$. In the corresponding segment, the water depth is constant. The analysis was performed in the following order: (1) Data 

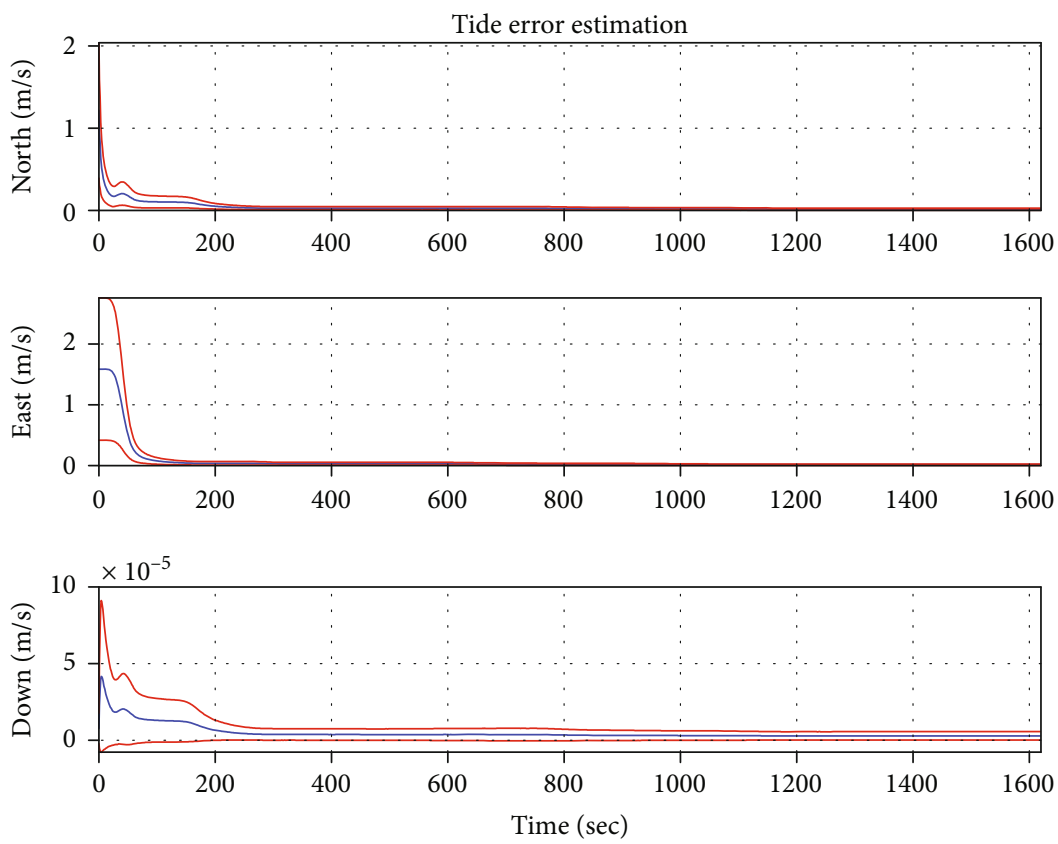

- Estimation result

Figure 8: Results of tide error estimation.
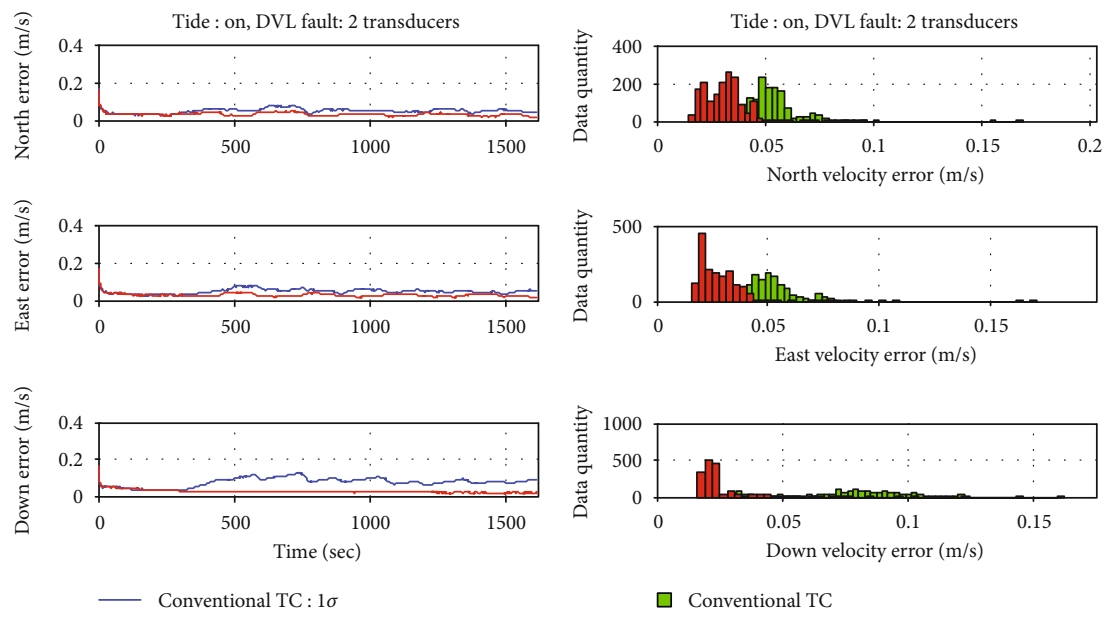

$\square$ Conventional TC

— Proposerd TC: $1 \sigma$

$\square$ Proposed TC

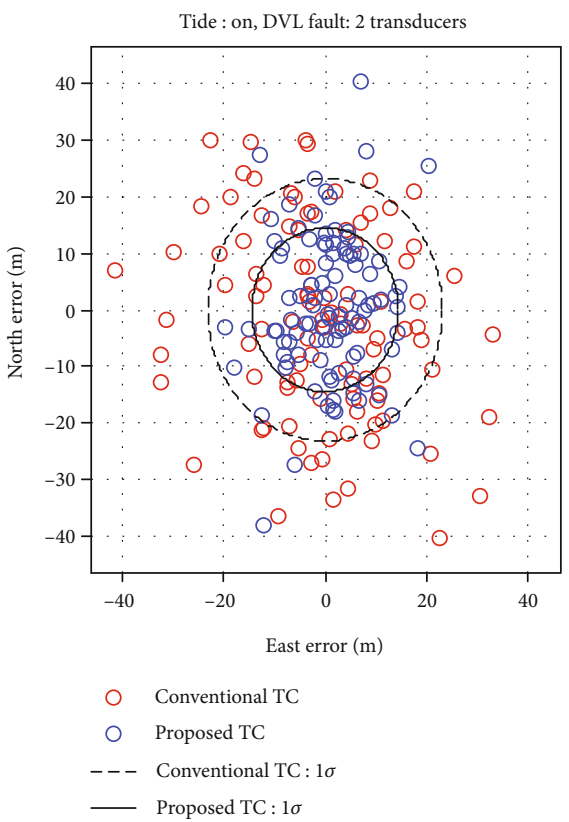

Figure 9: Result of Monte Carlo simulation (tide: on, DVL fault: two transducers).

for real sea testing was taken from an INS/DVL integrated navigation system with no history of DVL failure. The position error is denoted $\alpha$, as it is confidential information and cannot be disclosed. (2) DVL failure is implemented $5 \mathrm{~min}$ after navigation begins, in the same failure modes as in simulations. (3) The final position errors of the conventional and proposed filters are denoted " $\alpha+$ position error." That is, the position error is estimated with respect to the position error in a faultless condition.

Figure 12 presents real DVL outputs that verify the existence of tidal currents during the sea test. The water depth is constant in the analysis; therefore, transducers \#1 and \#3 are related to the $v_{x}^{b}$ component and transducers \#2 and \#4 are related to the $v_{y}^{b}$ component, as per Equation (11). In the 

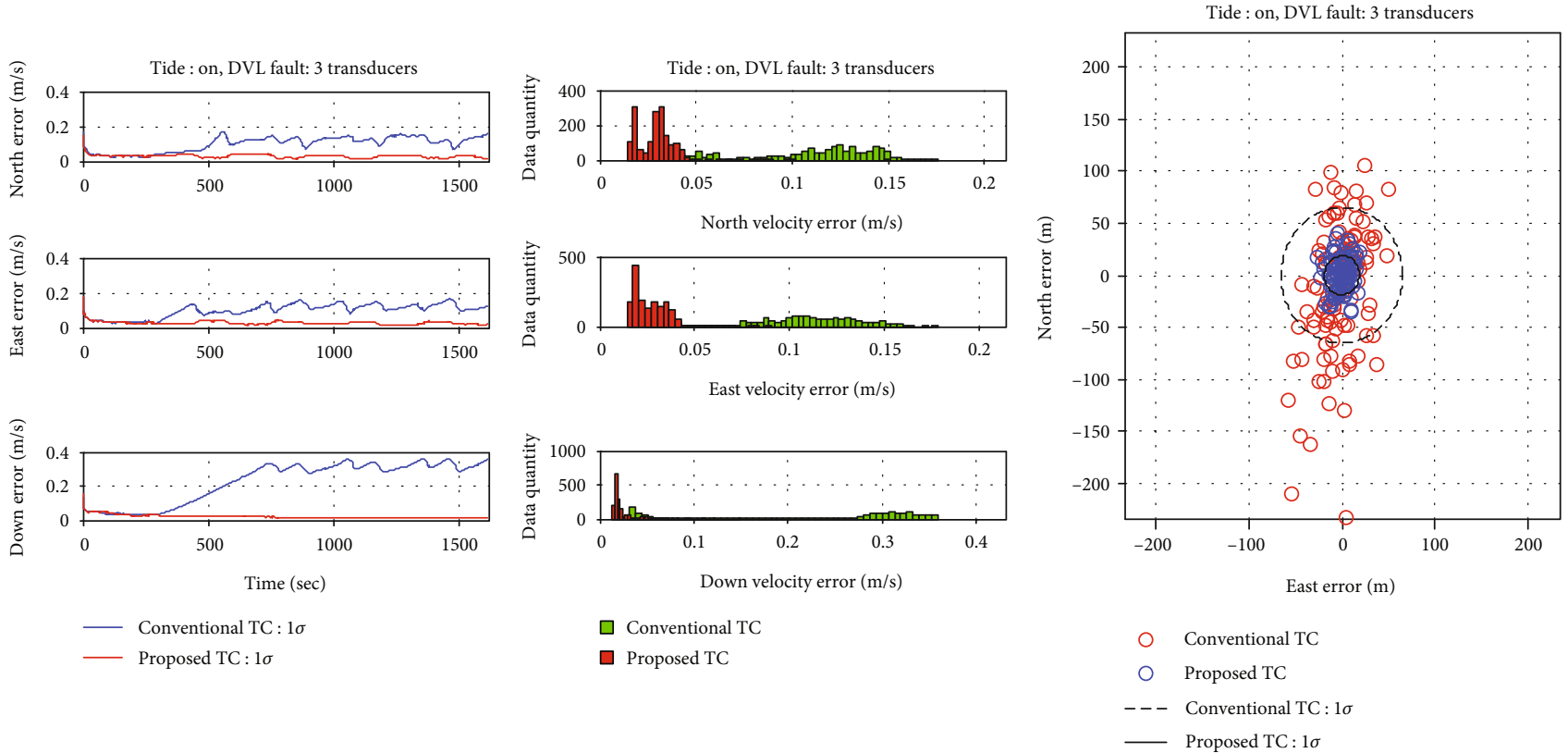

FIgURE 10: Result of Monte Carlo simulation (tide: on, DVL fault: three transducers).
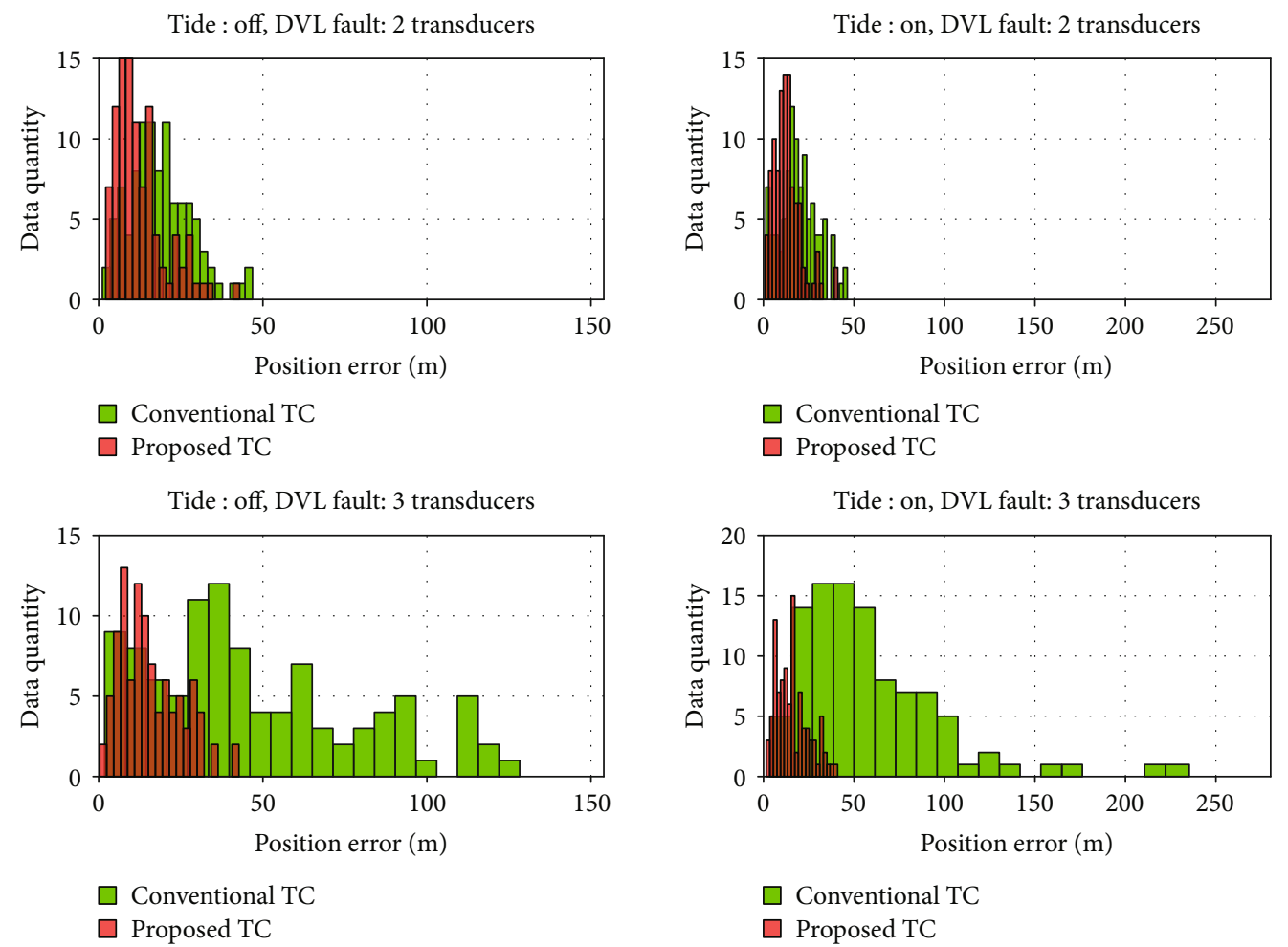

Figure 11: Position comparison in the Monte Carlo simulation result.

condition in which tidal currents are absent, the outputs of \#1 and \#3 must be constant and those of \#2 and \#4must be 0 . The existence of tide can be confirmed by the third graph in Figure 13, which depicts yaw angle information; it shows that DVL output changes whenever the yaw angle changes by 90 degrees. This can be determined to be the effect of tidal currents.
Figure 14 presents the real RPM data. In Figure 13, which illustrates the tide estimation results, the blue, red, and black lines represent the estimation results under conditions in which zero, two, and three DVL transducers are faulty, respectively. In real sea testing, it is difficult to predict the direction and speed of the effect of tidal currents. The blue line suggests that tidal information is estimated; however, 
TABLE 1: Result of position error estimation.

\begin{tabular}{|c|c|c|c|c|c|c|}
\hline \multirow[t]{2}{*}{ Case } & \multicolumn{2}{|c|}{ Tide } & \multicolumn{2}{|c|}{$\begin{array}{l}\text { DVL fault: number } \\
\text { of transducers }\end{array}$} & \multicolumn{2}{|c|}{ Position error $(\mathrm{m}, 1 \sigma)$} \\
\hline & Off & On & 2 & 3 & Conventional TC & Proposed TC \\
\hline 1 & $\mathrm{O}$ & - & - & - & 12.4965 & 12.7544 \\
\hline 2 & $\mathrm{O}$ & - & $\mathrm{O}$ & - & 22.1219 & 14.5247 \\
\hline 3 & $\mathrm{O}$ & - & - & $\mathrm{O}$ & 59.3077 & 18.6338 \\
\hline 4 & - & $\mathrm{O}$ & - & - & 14.7738 & 14.8691 \\
\hline 5 & - & $\mathrm{O}$ & $\mathrm{O}$ & - & 23.1579 & 14.9494 \\
\hline 6 & - & $\mathrm{O}$ & - & $\mathrm{O}$ & 65.0782 & 18.7233 \\
\hline
\end{tabular}

Velocity at DVL frame $(\mathrm{m} / \mathrm{s})$
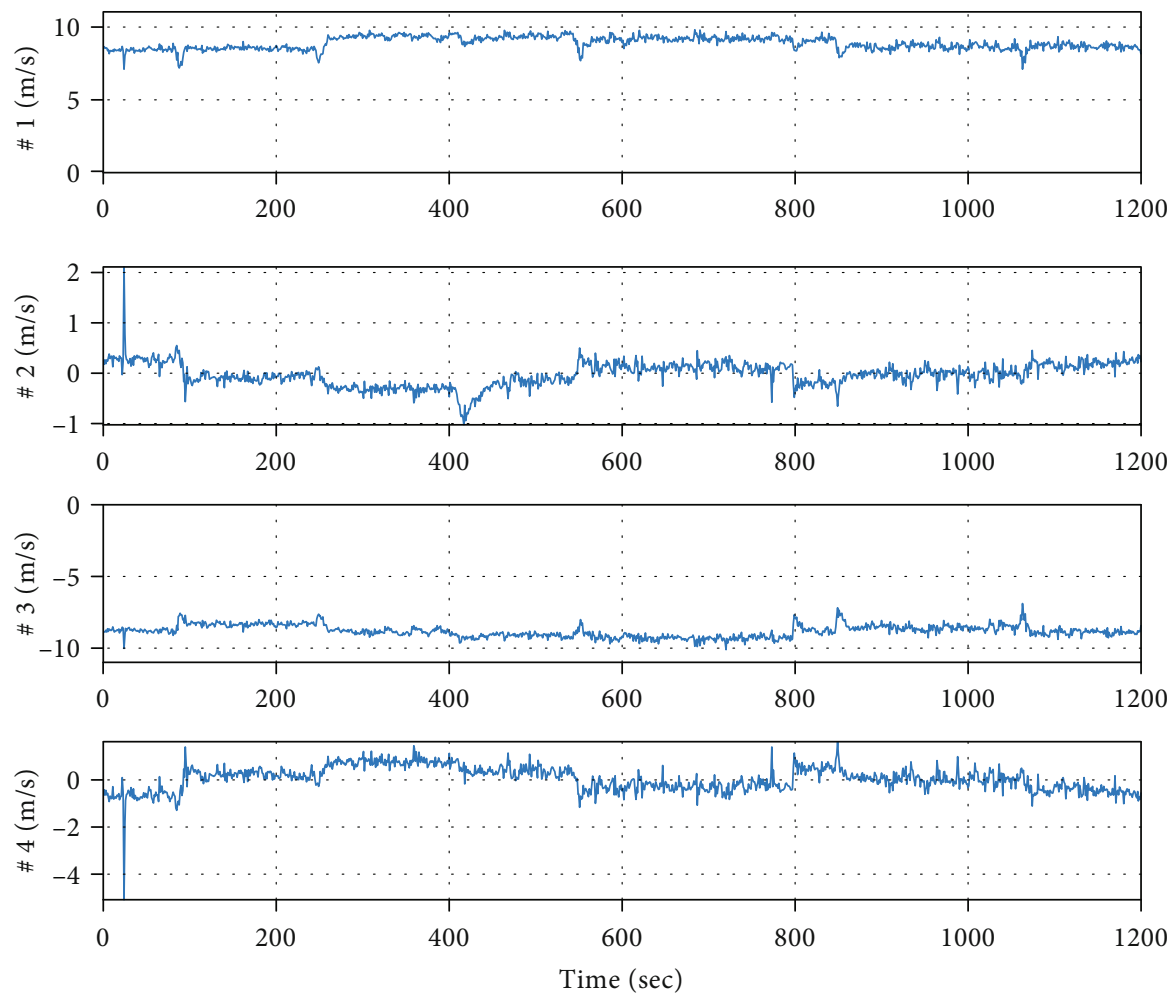

Figure 12: Experimental DVL data measurements.

more importantly, the red line shows that error estimation is carried out constantly based on the data from any normally functioning transducers despite the failure of two transducers. Error estimation is also performed, albeit to a lesser degree, even in the condition in which three transducers are faulty. This reaffirms the superiority of the proposed filter. Furthermore, a comparison with the yaw angle graph reveals that tide estimation accuracy increases when the yaw angle changes. This is because the reference frame used for tide error modeling is the navigation frame. Figures 15 and 16 show the velocity error results in DVL failure conditions. The green, blue, and red graphs represent conditions in which the DVL is intact, the conventional tightly coupled method, and the proposed tightly coupled method, respectively. As in the simulation results, the proposed method outperforms the conventional method in estimating velocity error. The accuracy of the estimated velocity error decreases as the number of faulty transducers increases in the conventional method whereas there is little or no difference in the proposed method even if the number of faulty transducers increases. The final position errors are given in Table 2.

\section{Conclusions}

This paper described the design of a tightly coupled INS/DVL/RPM integrated navigation system. We designed a filter that reproduces the measurements of a faulty transducer based on the advantages of the conventional tightly coupled integration method. The measurements reproduced 
Tide estimation result
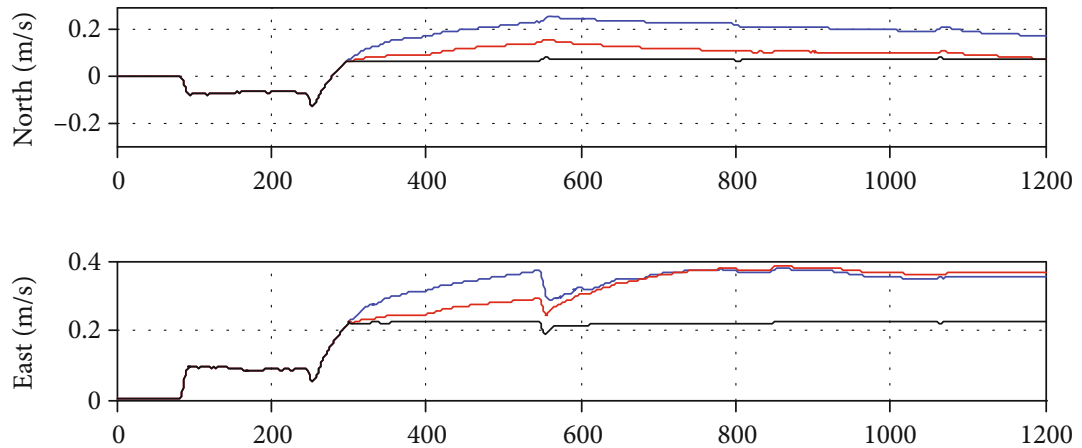

Attitude - yaw

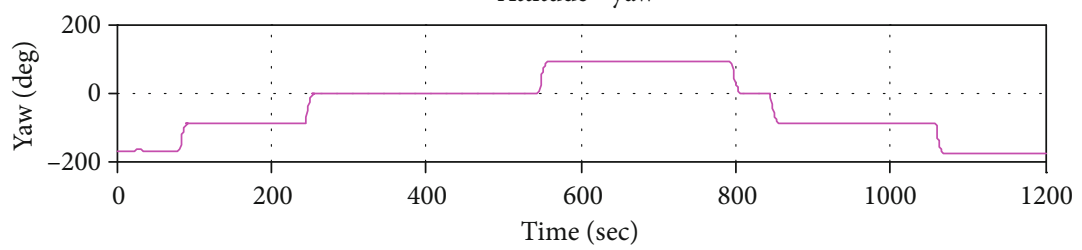

C DVL fault : no

— DVL fault : 2 transducers

_ DVL fault : 3 transducers

Figure 13: Results of tide estimations.

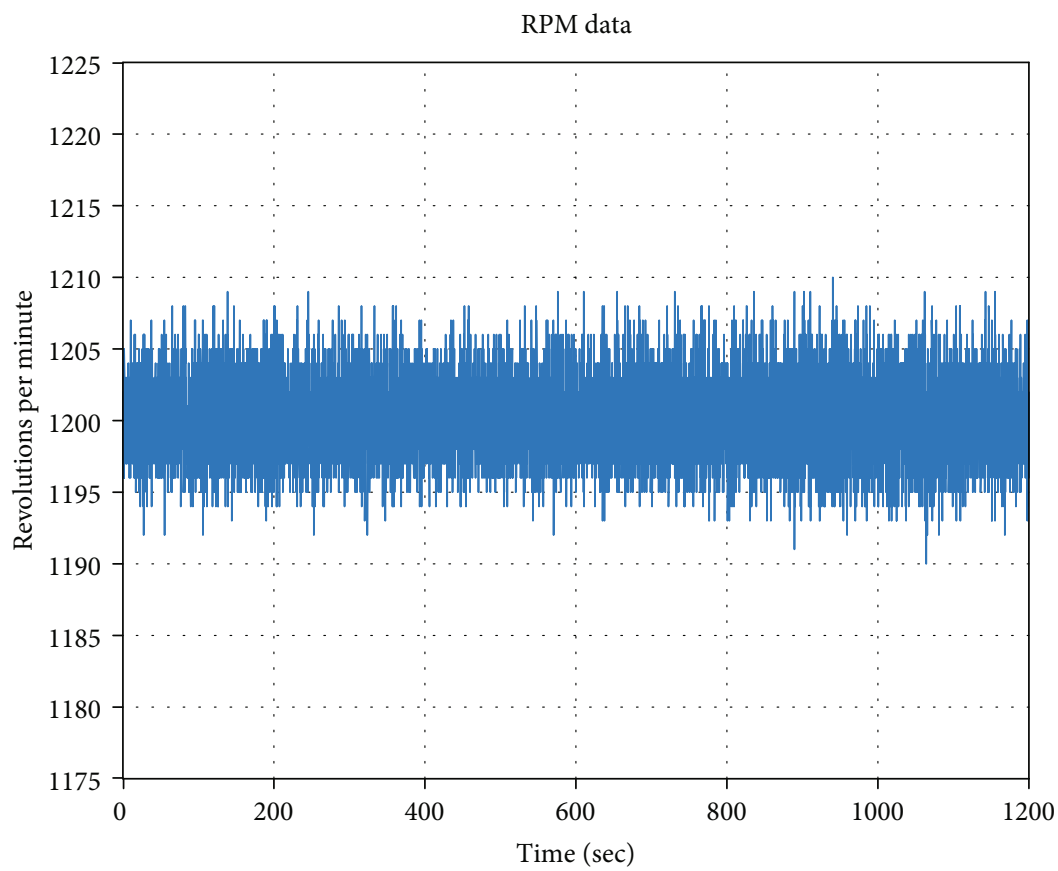

FIGURE 14: Experimental RPM data measurements.

were those taken by normally functioning DVL transducer and consisted of RPM-based estimates of velocity. In designing the filter, care was taken to ensure accurate RPM-based velocity estimation that took the effect of tidal currents into account. A salient feature of the proposed filter is that in the event of the failure of one DVL transducer, all measure- ments are not transformed into RPM; this is only done for the data from the faulty transducers. This allows a continuous use of measurements because the filter would continue to estimate RPM-based error. To verify the performance of the proposed filter, we conducted Monte Carlo simulations; analysis of their results revealed that the proposed filter could 

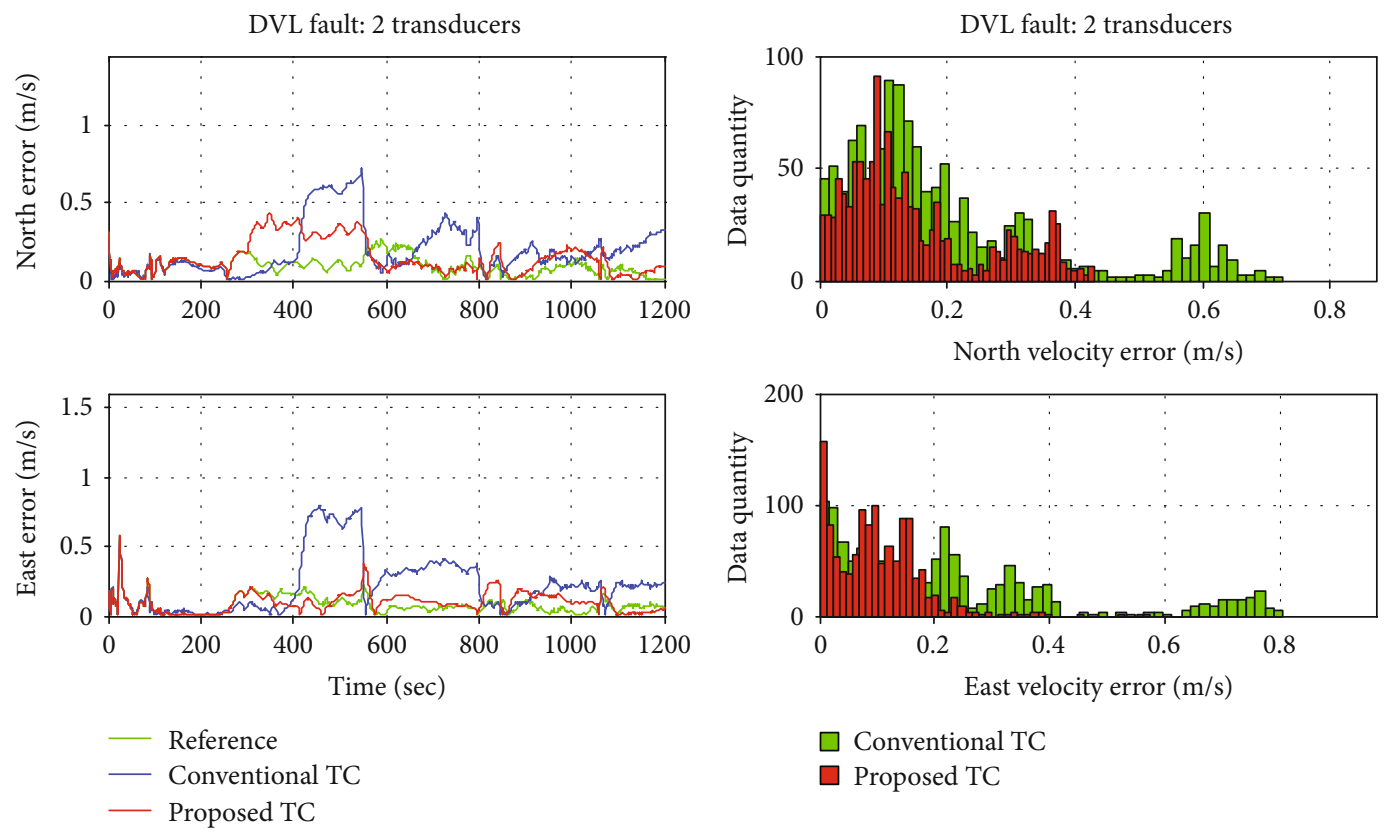

FIGURE 15: Result of velocity error estimations (DVL fault: two transducers).
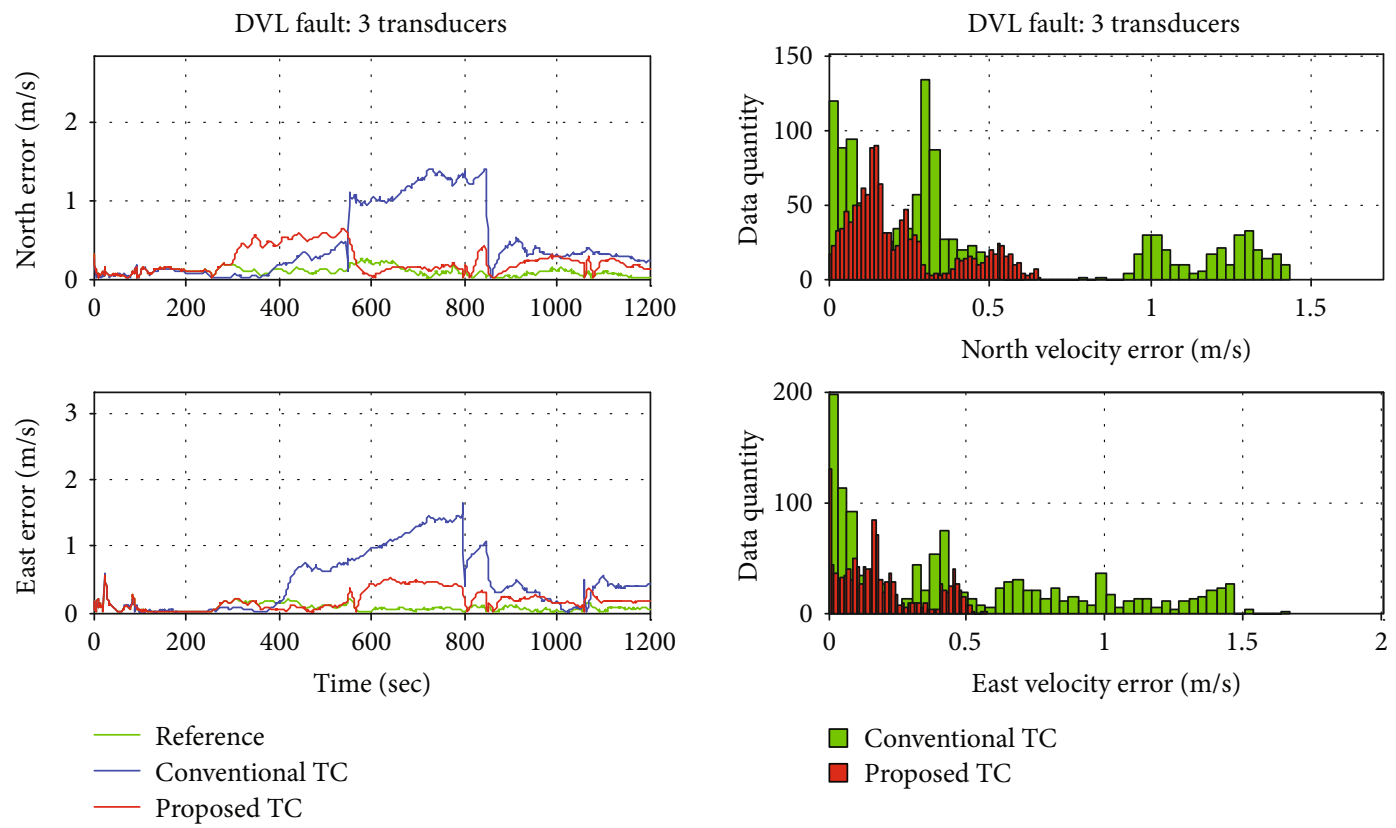

FIGURE 16: Result of velocity error estimations (DVL fault: three transducers).

TABLE 2: Result of position error.

\begin{tabular}{|c|c|c|c|c|}
\hline \multirow[t]{2}{*}{ Case } & \multicolumn{2}{|c|}{$\begin{array}{l}\text { DVL fault: } \\
\text { number of } \\
\text { transducers }\end{array}$} & \multicolumn{2}{|c|}{ Position error $(\mathrm{m})$} \\
\hline & 2 & 3 & Conventional TC & Proposed TC \\
\hline 1 & $\mathrm{O}$ & - & $\alpha+96.07$ & $\alpha+30.23$ \\
\hline 2 & - & $\mathrm{O}$ & $\alpha+457.38$ & $\alpha+59.01$ \\
\hline
\end{tabular}

estimate velocity and position errors more accurately than the conventional tightly coupled method irrespective of the effect of tidal currents and transducer failure. Finally, we verified the performance of the proposed filter using real sea test data.

This study analyzed the performance of the proposed filter using DVL failure scenario simulations. However, transducer failures are not the only DVL-related variables. For example, abrupt changes in depth can reduce transducer measurement accuracy even if they are all intact. Likewise, DVL measurements may not be accurate in segments in 
which an underwater vehicle moves beyond the DVL measurement range due to the topography of the ocean floor. It is therefore necessary to conduct follow-up tests in which the proposed filter is applied to ensure robust velocity error estimation in such variable circumstances.

\section{Data Availability}

(1) The (detailed equation for model) data used to support the findings of this study are included within the article. (2) The (figures) data used to support the findings of this study are included within the article.

\section{Conflicts of Interest}

The authors declare that there is no conflict of interest regarding the publication of this paper.

\section{Acknowledgments}

We would like to thank Editage (http://www.editage.co.kr) for English language editing. This research was supported by a self-investment from the R\&D Program in underwater navigation system funded by the Maritime R\&D Laboratory, LIGNex1 Co., Ltd.

\section{References}

[1] D. Titterton and J. Weston, Strapdown Inertial NavigationTechnology, Lavenham Press, London, UK, 2nd edition, 2004.

[2] D. Rudolph and T. A. Wilson, "Doppler Velocity Log theory and preliminary considerations for design and construction," in 2012 Proceedings of IEEE Southeastcon, pp. 15-18, Orlando, FL, USA, 2012.

[3] P. M. Lee, B. H. Jeon, S. M. Kim et al., "An integrated navigation system for autonomous underwater vehicles with two range sonars, inertial sensors and Doppler velocity log," in Oceans '04 MTS/IEEE Techno-Ocean '04 (IEEE Cat. No.04CH37600), pp. 1586-1593, Kobe, Japan, 2004.

[4] B. Jalving, K. Gade, K. Svartveit, A. Willumsen, and R. Sørhagen, "DVL velocity aiding in the HUGIN 1000 Integrated Inertial navigation system," Modeling, Identification and Control, vol. 25, no. 4, pp. 223-236, 2004.

[5] Ø. Hegrenæs and E. Berglund, "Doppler water-track aided inertial navigation for autonomous underwater vehicle," in In Proceedings of the IEEE Bremen: Balancing Technology with Future Needs (OCEANS '09), Bremen, Germany, 2009.

[6] X. Liu, X. Xu, Y. Liu, and L. Wang, "Kalman filter for crossnoise in the integration of SINS and DVL," Mathematical Problems in Engineering, vol. 2014, Article ID 260209, 8 pages, 2014.

[7] L. Stutters, H. Liu, C. Tiltman, and D. J. Brown, "Navigation technologies for autonomous underwater vehicles," IEEE Transactions on Systems, Man, and Cybernetics, Part C (Applications and Reviews), vol. 38, no. 4, pp. 581-589, 2008.

[8] L. Kang, L. Ye, and K. Song, "A Fast in-Motion Alignment Algorithm for DVL Aided SINS," Mathematical Problems in Engineering, vol. 2014, Article ID 593692, 12 pages, 2014.

[9] W. Gao, Y. Zhang, and J. Wang, "A Strapdown interial navigation system/Beidou/Doppler velocity log integrated navigation algorithm based on a cubature Kalman filter," Sensors, vol. 14, no. 1, pp. 1511-1527, 2014.

[10] X. Xu, P. Li, and J. Liu, "A fault-tolerant filtering algorithm for SINS/DVL/MCP integrated navigation system," Mathematical Problems in Engineering, vol. 2015, Article ID 581909, 12 pages, 2015.

[11] L. Paull, S. Saeedi, M. Seto, and H. Li, "AUV navigation and localization: a review," IEEE Journal of Oceanic Engineering, vol. 39, no. 1, pp. 131-149, 2014.

[12] L.-Y. Zhao, X. J. Liu, L. Wang, Y. H. Zhu, and X. X. Liu, “A pretreatment method for the velocity of DVL based on the motion constraint for the integrated SINS/DVL," Applied Sciences, vol. 6, no. 3, p. 79, 2016.

[13] Y. Kang, L. Zhao, J. Cheng, M. Wu, and X. Fan, "A novel grid SINS/DVL integrated navigation algorithm for marine application," Sensors, vol. 18, no. 2, p. 364, 2018.

[14] A. Tal, I. Klein, and R. Katz, "Inertial navigation system/Doppler velocity log (INS/DVL) fusion with partial DVL measurements," Sensors, vol. 17, no. 2, p. 415, 2017.

[15] P. Liu, B. Wang, Z. Deng, and M. Fu, "INS/DVL/PS tightly coupled underwater navigation method with limited DVL measurements," IEEE Sensors Journal, vol. 18, no. 7, pp. 2994-3002, 2018. 